\title{
CARDIOGENIC SHOCK IN PATIENT WITH ACUTE INSTALLATION PULMONARY HYPERTENSION
}

\begin{abstract}
Miriam Fang Castro ${ }^{1, \star}$, Guilherme Pereira Carlesso ${ }^{1}$, João Paulo Peres Lima ${ }^{1}$, Leonardo Afonso Costa ${ }^{1}$, Lucas de Castro Barroti ${ }^{1}$, Isadora Medina ${ }^{1}$, Izaura Tereza Silva Guedes ${ }^{1}$, Rodrigo Lorenzetti Serrano ${ }^{1}$, Renan Rodrigues Neves Ribeiro do Nascimento ${ }^{1}$, Edgard Torres dos Reis Neto ${ }^{1}$, Isaac Felipe Leite Braz ${ }^{1}$
\end{abstract}

1.Universidade Federal de São Paulo, São Paulo (SP), Brazil.

*Corresponding author: miriamcfang@gmail.com

\section{BACKGROUND}

Antisynthetase syndrome (ASS) is an autoimmune disease characterized by interstitial lung disease (ILD), inflammatory arthritis and myopathy, skin hyperkeratosis (mechanic's hands) and Raynaud's phenomenon as the main symptoms. The lung is the most involved extramuscular organ in ASS and it defines the course of the disease, with pulmonary arterial hypertension (PAH) being a rare finding and without an established screening. Anti-aminoacyl-tRNA-synthetase (anti-ARS) autoantibodies have been correlated with specific clinical presentations and prognosis.

\section{CASE REPORT}

A 24-year-old white woman, with chronic neutrophilic urticaria for 2 years, was hospitalized with asthenia, fever and dyspnea at rest for 6 days. She reported progressive 5-month-onset dyspnea, unintentional weight loss of $10 \mathrm{~kg}$, heartburn, small-large joint polyarthralgia and extremity cyanosis. At admission, she presented tachycardia, tachypnea, hypoxemia, pulmonary rales, left knee and 3rd right proximal interphalangeal arthritis and toes desquamation. The patient progressed to respiratory failure and cardiogenic shock. Laboratory examination revealed Hb $11 \mathrm{~g} / \mathrm{dL}$, erythrocyte sedimentation rate $15 \mathrm{~mm} / \mathrm{h}$, high creatine kinase 6600 $\mathrm{U} / \mathrm{L}$, troponin elevation from 78 to $257 \mathrm{pg} / \mathrm{mL}$. Point-of-care echocardiography displayed a dilated right ventricle with decreased contractility and area fractional variation of 20\% (FAC normal range $>35 \%$ ). Other cavities with normal dimensions and preserved left ventricular function. Pulmonary computed tomography (CT) angiography ruled out venous thromboembolism and identified ground-glass and reticulate opacities predominantly peripheral in the lower lobes, with some traction bronchiectasis and subpleural cysts, findings compatible with nonspecific interstitial pneumonia. Computed tomography scan also showed esophageal dilation. To investigate the etiology of elevated muscle and cardiac enzymes, cardiac magnetic resonance imaging was performed, which did not reveal signs of myocarditis or ischemic involvement. The patient recovered after using dobutamine and norepinephrine, volume management and starting prednisone $1 \mathrm{mg} / \mathrm{kg} /$ day. After clinical stabilization, she performed right cardiac catheterization, which confirmed precapillary pulmonary hypertension with pulmonary vascular hyperresistance, in addition to maintenance of echocardiographic and tomographic parameters. The myopathy panel revealed positivity for anti-Ro52 and anti-PL12. Once the diagnosis of ASS was defined, the patient received high dose corticosteroid pulse therapy and mycophenolate sodium due to the severe involvement of the disease.

\section{CONCLUSION}

Pulmonary hypertension is an uncommon manifestation in ASS, usually associated with ILD. In this case, we observed a disproportion of pulmonary parenchyma and arterial findings in a patient with acute and progressive development of secondary cardiac involvement. This report shows that early recognition and proper management of connective tissue disease-related PAH is critical to successful outcome.

\section{KEYWORDS}

Antisynthetase syndrome, Pulmonary arterial hypertension, Cardiogenic shock. 